\title{
Direct and converse magnetoelectric effect at resonant frequency in laminar piezoelectric-magnetostrictive composite
}

\author{
C. Popov ${ }^{2}$, H. Chang ${ }^{1}$, P.M. Record ${ }^{2}$, E. Abraham ${ }^{2}$, R.W. Whatmore ${ }^{1,3}$ and Z. Huang ${ }^{1, *}$ \\ ${ }^{1}$ Department of Materials, School of Applied Science, Cranfield University, Beds, MK43 OAL, UK \\ ${ }^{2}$ School of Engineering and Physical Sciences, Heriot-Watt University, Edinburgh EH14 4AS, UK \\ ${ }^{3}$ Current Address: Tyndall National Institute, Cork, Ireland. \\ *Corresponding author e-mail: Z.Huang@cranfield.ac.uk
}

\begin{abstract}
Laminar piezoelectric-magnetostrictive composites using piezoelectric lead zirconate titanate ceramics and the giant magnetostrictive rare-earth-iron alloy Terfenol-D were prepared by epoxy bonding. The direct and converse magnetoelectric (ME) effects at and off the mechanical resonant frequency were characterized and compared to the theoretical modelling. The mechanical resonant frequency of the composites depended on the sample orientation and the magnetic DC bias field. In the longitudinal configuration, the resonant frequency shifted down monotonically with the increasing bias field. When the sample was in the transverse configuration, the resonant frequency decreased with the increasing field at first. However, at higher bias, it shifted up with the increasing bias. A phenomenological model based on the $\Delta \mathrm{E}$ effect of magnetostrictive materials is proposed to explain the observed phenomena.
\end{abstract}

Keywords: magnetoelectric effect, piezoelectric, magnetostrictive, PZT, $\Delta \mathrm{E}$ effect, orientation dependence. 


\section{Introduction}

The fact that the magnetisation of a magnetic material can be controlled by an external magnetic field is widely used in today's information technology. The manipulation of magnetisation by means other than magnetic field, for example electric field, could find applications in magnetic storage, spintronics [1], and other magnetic sensing and actuating micro-electric-mechanical systems (MEMS) devices [2]. Electrically-controlled magnetic switches have been reported recently in the hole-induced ferromagnetic semiconductor (In,Mn)As [3] and the magnetic ferroelectric compound $\mathrm{HoMnO}_{3}$ [4] via magnetoelectric interaction. However, both of these were observed at very low temperatures (less than $25 \mathrm{~K}$ ). Recently, electrically tunable ferromagnetic resonance [5,6] via magnetoelectric (ME) interaction and the converse ME effect at low frequency [7] have been reported.

The ME effect is characterized by the appearance of an electric polarization on applying a magnetic filed ( $\alpha=\delta \mathbf{P} / \delta \mathbf{H}$, called the $\mathrm{ME}_{\mathrm{H}}$ effect, or direct ME effect) or by the appearance of a magnetization on applying an electric field $\left(\alpha=\delta \mathbf{M} / \delta \mathbf{E}\right.$, called $\mathrm{ME}_{\mathrm{E}}$ effect, or converse ME effect). It was foreseen by Landau and Lifshitz from thermodynamics and symmetry consideration [8] and was first measured in a $\mathrm{Cr}_{2} \mathrm{O}_{3}$ crystal $[9,10]$. The $\mathrm{ME}$ effect in single phase materials is usually very small and often can only be realised at very low temperatures $[11,12]$.

The ME effect can also be realised through product property [13]. Van Suchtelen proposed the product property concept whereby entirely new properties could be achieved by coupling a pair of tensor properties in two materials that were intimately mixed [13]. Since then two main processing routes have been investigated to achieve ME effect this way, namely, (a) particulate magnetostrictive (MS) and piezoelectric (PE) and (b) laminated MS/PE composites. Investigations on eutectic co-existing phases of spinel cobalt ferrite-cobalt titanate and perovskite barium titanate 
achieved magnetoelectric voltage coefficient $\alpha_{\mathrm{E}}=\alpha / \varepsilon_{0} \varepsilon_{\mathrm{r}} \sim 50 \mathrm{mV} / \mathrm{cm}$ Oe despite theoretical estimation to be $40-60$ times larger [14], where $\varepsilon_{\mathrm{r}}$ is the relative permittivity and $\varepsilon_{0}$ the dielectric constant of vacuum. Similar ME coefficient were obtained with particulate composites of the giant MS rare-earth-iron alloy $\mathrm{Tb}_{1-\mathrm{x}} \mathrm{Dy}_{\mathrm{x}} \mathrm{Fe}_{2}$ (TFD) and lead zirconate titanate (PZT) and PVDF polymer [15]. A recent investigation of $\mathrm{CoFe}_{2} \mathrm{O}_{4}-\mathrm{BiFeO}_{3}$ nanocomposites obtained $\alpha_{\mathrm{E}}$ as high as $280 \mathrm{mV} / \mathrm{cm}$ Oe [16]. On the other hand, laminated MS/PE structures have been found to show much higher $\mathrm{ME}_{\mathrm{H}}$ coefficients. $\alpha_{\mathrm{E}}$ values of $1500 \mathrm{mV} / \mathrm{cm}$ Oe for ferrite-PZT [17] and $4680 \mathrm{mV} / \mathrm{cm}$ Oe for TFD/PZT laminar structures [18] have been reported. Significant enhancement of $\mathrm{ME}_{\mathrm{H}}$ response can be obtained when the modulation frequency of the applied fields coincides with magnetic, electrical or mechanical eigenmodes of the system under study [19-21]. Here, we report the investigation of the direct and converse ME effects in composites at and off their mechanical resonant frequencies and the comparison between these measurement results and the theoretical modelling.

\section{Experimental}

The giant magnetostrictive rare-earth-iron alloy TFD (Gansu Tianxing Rare Earth Functional Materials Co, China) and the piezoelectric PZT ceramics (Ferroperm Piezoceramics, PZ29) were used to make laminar sandwich structures TFD/PZT/TFD and PZT/TFD/PZT by epoxy bonding ( EPOTEK 301-2) at $85^{\circ} \mathrm{C}$. Meso-scale samples, with total thickness of $1.262 \mathrm{~mm}$ were fabricated by polishing down the TFD and then cutting the laminated composites. The PZT thickness was 0.127 $\mathrm{mm}$, and the epoxy layer thickness was about $0.01 \mathrm{~mm}$. The laminar was suspended between two thin $\mathrm{Cu}$ wires attached to the face centres. These two wires also served as two electrodes. This mounting allowed minimum physical clamping on the sample. 
The direct ME effect was measured using an AC magnetic field generated from a Helmholtz coil as input and measure the induced electric voltage across the PZT as the output. The characterisation of the converse ME effect, namely, the electric voltage applied to the PZT as the input and the induced magnetisation of the TFD as the output, was carried out using a set-up similar for the direct ME measurement, without the Helmholtz coil. A sinusoidal voltage was applied to the PZT, and the induced AC magnetic field was measured by a pickup coil placed immediately outside the sample. Two pickup coils placed at perpendicular directions at the same time were used for the three dimensional characterisation of the induced magnetisation field. The DC bias magnetic fields were provided by a pair of electromagnet. For all measurements presented here, the AC and DC magnetic fields were parallel. At high frequency the inductance of the Helmholtz coil generates a prohibitively large voltage so for frequencies above $10 \mathrm{kHz}$ the inductance was tuned out by a series variable capacitance. The total capacitance required to put the coils in resonance was calculated from the inductance and resistance measured with RCL Bridge (Fluke, PM6306). For frequencies below $10 \mathrm{kHz}$ the Helmholtz coil pair was driven directly. More experimental details can be found elsewhere [22]. The longitudinal and transverse measurement configurations have been investigated, as schematically shown in Fig. 1.

\section{Results and Discussion}

Fig. 2a shows typical ME response as a function of frequency at transverse configuration. At low frequencies $(<40 \mathrm{KHz})$ the ME voltage coefficient $\alpha_{\mathrm{v}}=\mathrm{E} / \mathrm{H}$ was almost a constant. The $\alpha_{\mathrm{v}}$ was found to depend on the DC bias field, and had the highest value for the bias field around 400 Oe. This is understandable since the ME coefficient is proportional to the MS coefficient $\mathrm{q}_{31}$, and $\mathrm{q}_{31}$ reaches its maximum at $\mathrm{H}_{\mathrm{dc}}$ around 350 Oe [23]. The $\alpha_{\mathrm{v}}$ had a sharp increase around the resonant frequency. The nature of this resonance was dominated by mechanical effect, as can be confirmed 
from the following calculation: Let $\mathrm{f}_{\mathrm{r}}$ be the fundamental mechanical resonant frequency of the bonded structure, and assuming the bonding is perfect and had negligible thickness, then [24]

$$
f_{r}=\frac{1}{2 l} \sqrt{\frac{v_{P Z T} Y_{p Z t}+v_{t f d} Y_{t f d}}{\rho_{P Z T} v_{P Z T}+\rho_{t f d} v_{t f d}}}
$$

Here $l=14.5 \mathrm{~mm}$ was the sample length. Using material parameters Young's modulus $Y_{t f d}=4 \times 10^{10}$ $\mathrm{Nm}^{-2}, Y_{p z t}=5.9 \times 10^{10} \mathrm{Nm}^{-2}$, volume fraction $v_{t f d}=0.9, v t_{p z t}=0.1$, density $\rho_{t f d}=9.2 \mathrm{Kg} \mathrm{m}^{-3}$ and $\rho_{p z t}=7.46 \mathrm{Kg} \mathrm{m}^{-3}$ it can be obtained $\mathrm{f}_{\mathrm{r}}=74.3 \mathrm{KHz}$, which agrees well with the observed values. The peak height of the $\alpha_{\mathrm{v}}$ at resonance was found to depend on the DC and AC magnetic fields, the sample orientation, as well as the particular sample (Fig.2b, another sample), a value up to $\alpha_{\mathrm{v}}=90 \mathrm{~V}$ $\mathrm{cm}^{-1} \mathrm{Oe}^{-1}$ has been observed at the resonance. The linearity between the voltage output across the PZT and the magnetic AC field applied to the TFD has been confirmed for both the low frequency and resonant responses. Unlike the low frequency response, the $\alpha_{\mathrm{v}}$ at resonance was found to increase with the bias field up to $\mathrm{H}_{\mathrm{dc}}=1000 \mathrm{Oe}$, which was the maximum bias field used in this experiment. The $\alpha_{\mathrm{v}}$ was measured as $11.3 \mathrm{~V} \mathrm{~cm}^{-1} \mathrm{Oe}^{-1}$ at $70 \mathrm{KHz}$ at the DC bias 300 Oe. From Fig. 1a curve's behaviours this suggested $\alpha_{\mathrm{v}}$ about $11.3 / 3=3.8 \mathrm{~V} \mathrm{~cm}^{-1} \mathrm{Oe}^{-1}$ at low frequency $(<40 \mathrm{KHz})$. Using equation (13) in Ref 25 and material parameters listed in the Table I, the calculated ME voltage coefficient is $\alpha_{\mathrm{v}}=8.2 \mathrm{~V} \mathrm{~cm}^{-1} \mathrm{Oe}^{-1}$ which agrees well with the measured value.

Similar to the direct ME effect, the converse ME response was nearly a constant at low frequency $(<40 \mathrm{KHz})$, and the ME Oersterd coefficient [26] $\alpha_{\mathrm{H}}=\mathrm{M} / \mathrm{E}$ was measured as 9 Oe $\mathrm{cm} / \mathrm{KV}$ (assuming $\mu_{\mathrm{r}}=1$ ) at $70 \mathrm{KHz}$ for a bias field of $300 \mathrm{Oe}$, here $\mathrm{M}$ is the induced magnetisation in the TFD and E the electric field across the PZT. The $\alpha_{H}$ also peaked at the resonance frequency (79KHz) with a value of $50 \mathrm{Oe} \mathrm{cm} / \mathrm{KV}$ (Fig. 3). The theoretically calculated value is $19 \mathrm{Oe} \mathrm{cm} / \mathrm{KV}$ at low frequency (well below resonance) if the bonding is assumed to be perfect [26]. Both the 
calculated and the measured values were more than 3 orders of magnitude larger than the value for $\mathrm{Cr}_{2} \mathrm{O}_{3}$ for which the $\alpha_{\mathrm{H}}$ is no more than $41 \times 10^{-4} \mathrm{Oe} \mathrm{cm} / \mathrm{KV}$.

Another interesting feature of the ME effect is the shift of the resonant frequency with the applied magnetic bias field. Fig. 4 and Fig. 2 show the resonant frequency dependence on the bias field for (a) the longitudinal and (b) transverse configurations for different AC fields respectively. In the longitudinal configuration, the sample's length, which was also the easy axis of the TFD was perpendicular to the $\mathrm{DC}$ and $\mathrm{AC}$ magnetic fields, the resonant frequency shifted lower monotonically with the increasing bias field (Fig. 5a). When the sample was in the transverse configuration, the sample's length and therefore the easy axis was parallel to the DC and AC magnetic fields. At small DC bias field, the resonant frequency decreased with the increasing field. However, at higher bias, it shifted up with the increasing bias (Fig. 5b). For the converse ME response (Fig. 3), the resonant frequency shift as a function of the magnetic bias field was similar to those observed in the direct ME effect. This dependence of resonant frequency on the bias magnetic field is consistent with the $\Delta \mathrm{E}$ effect, which is the dependence of the Young's modulus of the MS materials on the magnetic field. For MS materials, the strain produced by the MS effect adds to the stress-induced strain, so the material becomes softer when the magnetic moments are free to rotate. It becomes increasingly stiff when approaching saturation and magnetic moment mobility decreases. Squire [27] developed a phenomenological model for the $\Delta \mathrm{E}$ effect and we use this model to explain the above observed results.

In eqn (1), $Y_{p z t}$ and $\rho_{p z t}$ does not change with magnetic field since the PZT is not a magnetic material, and $v_{t f d}, v_{p z t}$ and $\rho_{t f d}$ do not change much since the volume change with magnetic field is close to zero. Therefore, we can discuss the dependence of $Y_{t f d}$ on the magnetic field directly. According to Squire [27], $\frac{Y}{Y_{s}}=\frac{1}{1+\left(9 \lambda_{s}^{2} Y_{s} / 8 K\right) F(h, \theta, \gamma)}$, here $\lambda_{s}, Y_{s}$ and $K$ are respectively the MS coefficient at saturation, Young's modulus at saturation and anisotropy energy density of the TFD. 
$F(h, \theta, \gamma)$ is a function of the reduced magnetic field $h=H \mu_{0} M_{s} / 2 K$, reduced strain energy $\gamma=3 \lambda_{s} \sigma / 4 K$, and wall angle $\theta$ between the easy axis and the external field direction. At the longitudinal configuration, $\theta=90^{\circ}$, the magnetisation is by means of moment rotation only and its Young's modulus decreases monotonically until saturation at $h=1$. At the transverse configuration, $\theta=0^{\circ}$, the magnetisation is by means of domain wall movement only and its Young's modulus increases monotonically with the increasing bias field. Squire's model has singularity at $\theta=0^{\circ}$ so a small $\theta$ (e.g. $15^{\circ}$ ) is used for the numerical calculation. Fig. 5c depicts the calculated $Y_{t f d} / Y_{s}$ as functions of the reduced bias field for $\theta=90^{\circ}$ (solid line) and $\theta=15^{\circ}$ (dotted line). For our sample, the TFD was most likely to be composed of both the $\theta=90^{\circ}$ and $\theta=0^{\circ}$ domains at both the transverse and longitudinal configurations, due to the existence of an internal stress developed during the cooling of the sandwiched structure to room temperature from the curing temperature $\left(85^{\circ} \mathrm{C}\right)$. TFD has a much larger thermal expansion coefficient than the PZT, so at room temperature the TFD was under tensile and the PZT under compressive stresses. Assuming $\alpha$ is the thermal expansion coefficient, then the stress:

$$
\sigma=\frac{t_{p z t} Y_{p z t} * Y_{t f d}}{\left(t_{t f d} Y_{t f d}+t_{p z t} Y_{p z t}\right)} *\left(\alpha_{p z t}-\alpha_{t f d}\right) *\left(T_{2}-T_{1}\right)
$$

using $\alpha_{t f d}=11 \times 10^{-6} \mathrm{~K}^{-1}, \alpha_{p z t}=2 \times 10^{-6} \mathrm{~K}^{-1}, Y_{t f d}=4 \times 10^{10} \mathrm{Nm}^{-2}, Y_{p z t}=5.9 \times 10^{10} \mathrm{Nm}^{-2}, t_{t f d}=1.12 \mathrm{~mm}$,

$t_{p z t}=0.127 \mathrm{~mm}, T_{2}=85^{\circ} \mathrm{C}, T_{1}=20{ }^{\circ} \mathrm{C}$, we obtain $\sigma=3.3 \times 10^{6} \mathrm{Nm}^{-2}$. The stress induced magnetisation due to the Villari effect in the TFD can then be obtained from $B_{j}=q_{i j} T_{j}$. From the manufacturer's data curve, this corresponds to $13.5 \mathrm{KA} / \mathrm{m}=170$ Oe. The measured value $(85 \mathrm{Oe})$ agrees well with the above calculation. If we assume there were $80 \%$ and $20 \%$ of the $\theta=90^{\circ}$ and $\theta=0^{\circ}$ domains in the longitudinal configuration and $20 \%$ and $80 \%$ in the transverse configuration, respectively, the calculated $Y_{t f d} / Y_{s}$ as functions of the reduced bias field are depicted in the Fig. $5 \mathrm{c}$ as the dashed line and broken line respectively. These two profiles agree qualitatively well with our experimental observations.

\section{Summary}

Laminar piezoelectric-magnetostrictive composites using piezoelectric lead zirconate titanate and the giant magnetostrictive rare-earth-iron alloy Terfenol-D were prepared using epoxy bonding. The 
direct and converse magnetoelectric (ME) effects at and off the mechanical resonant frequency were characterized and compared to theoretical modelling. The mechanical resonant frequency of the composites was observed to depend on the sample orientations as well as the magnetic DC bias field. In the longitudinal configuration, the magnetic easy axis of the TFD was perpendicular to the DC and AC magnetic fields, the resonant frequency shifted down monotonically with the increasing bias field. When the sample was in the transverse configuration, the easy axis of the TFD was parllel to the DC and AC magnetic fields, the resonant frequency decreased with the increasing field initially. However, at higher bias field, it shifted up with the increasing bias. A phenomenological model based on $\Delta \mathrm{E}$ effect of magnetostrictive materials is proposed to explain the observed phenomena.

\section{Acknowledgement}

This work was financially supported by the UK EPSRC Grants GR/R92448, EP/D506638/1 and EP/C519426/1.

\section{References}

[1] G. A. Prinz, Science, 282, 1660-1663 (1998).

[2] M. Fiebig, J. Phys.D: Appl. Phys. 38, R123-152 (2005).

[3] H. Ohno, D. Chiba, F. Matsukura, T. Omiya, E. Abe, T. Diett, Y. Ohno, and K. Ohtani, , Nature 408, 944-946 (2000).

[4] T. Lottermoser, T. Lonkal, U. Amann, D. Hohlwein, J. Ihringer, and M. Fiebig, Nature 430, 541-544 (2004).

[5] A.A. Semenov, S.F. Karmanenko, V.E. Demidov, B.A. Kalinikos, G. Srinivasan, A.N. Slavin, and J.V. Mantese, Appl. Phys. Lett., 88, 033503 (2006). 
[6] Y. K. Fetisov and G. Srinivasan, Appl. Phys. Lett., 88, 143503 (2006).

[7] Y. Jia, S.W. Or, H.L.W. Chan, X. Zhao and H. Luo, Appl. Phys. Lett., 88, 242902 (2006).

[8] L.D. Landau and E. M. Lifshitz, "Electrodynamics of Continous Media," Pergamon Press, Oxford (1960) (Translation of Russian Edition, 1958).

[9] D.N. Astrov, Zh. Exp. Teor. Fiz., 37, 881 (1959) [Soviet Phys.-JETP, 10, 628 (1960)].

[10] V.J. Folen, G.T. Rado and E.W. Stalder, Phys. Rew. Lett., 6, 607 (1961).

[11] G.T. Rado, J.M. Ferrari, and W.G. Maisch, Phys. Rew. B29, 4041 (1984).

[12] K. Agyei and J. L. Birman, J. Phys.: Condens. Matter., 2, 3007 (1990).

[13] J. van Suchtelen, Philips Res. Rep., 27, 28 (1972).

[14] J. van den Boomgaard, D.R. Terrell, R.A.J. Born and H.F.J.I. Giller, J. Mater. Sci., 9, 1705 (1974); A.M.J.G. van Run, D.R. Terrell, and J.H. Scholing, J. Mater. Sci., 9, 1710 (1974).

[15] C.W. Nan, N. Cai, L. Liu, J. Zhai, Y. Ye and Y. Liu, J. Appl. Phys., 94(9), 5930 (2003).

[16] X. Liu, S. Fu and C. Huang, Mater. Sci. \& Engin., B121, 255 (2005).

[17] G. Srinivasan, E.T. Rasmussen, J. Gallegos, R. Srinivasan, Yu.I. Bokhan, and V.M. Laletin, Phys. Rev. B64, 214408 (2001)

[18] J. Ryu, A.V. Carazo, K. Uchino, and H.E. Kim, Jpn. J. Appl. Phys., Part 1, 40, 4948 (2001).

[19] U. Laletsin, N. Padubnaya, G. Srinivasan and C.P. Devreugd, Appl. Phys. A78, 33 (2004).

[20] S. Dong, J. Li, and D. Viehland, App. Phys. Lett. 83, 11 (2003).

[21] S. Dong, J. Li, and D. Viehland, App. Phys. Lett. 86, 182506 (2005).

[22] P.M. Record, C. Popov, E. Abraham, J. Fletcher, H. Chang, Z. Huang and R.W. Whatmore, submitted to Sensors and Actuators A-Physical.

[23] The magnetostriction $\left(\lambda_{/ /}\right)$data as supplied by the manufacturer as a function of magnetic field was approximated by a polynomial fitting, then its derivation which should equal the $\mathrm{q}_{33}$ was obtained. $\mathrm{q}_{31}$ was obtained by assuming $\mathrm{q}_{31}=-\mathrm{q}_{33} / 2$.

[24] S. Schmidt and C.A. Grimes, IEEE Trans. Mag. 37, 2731-2733 (2001).

[25] M.I. Bichurin and V.M. Petrov, Phys. Rew. B, 68, 054402 (2003). 
[26] Z. Huang, J. Appl. Phys, 100, 114104 (2006).

[27] P.T. Squire, J. Magn. Magn. Mat., 87, 299-310 (1990).

Figure captions

Fig. 1 Schematic of the configurations for (a) transverse; (b) longitudinal; and (c) converse ME effect measurements.

Fig. 2 Magnetoelectric voltage coefficients $\alpha_{v}$ as functions of frequency: (a) in the full frequency range $100-100 \mathrm{KHz}$ for one sample and (b) around resonant frequency for different DC bias fields for a transversely oriented TFD-PZT-TFD composite.

Fig. 3 Magnetoelectric Ostered coefficients $\alpha_{H}$ as functions of frequency for different DC bias fields for a transversely oriented TFD-PZT-TFD composite.

Fig. 4 Magnetoelectric voltage coefficients $\alpha_{v}$ as functions of frequency for different DC bias fields for a longitudinal oriented TFD-PZT-TFD composite.

Fig. 5 Magnetic bias field dependence of the resonant frequency for the (a) longitudinal and (b) transverse configuration with different ac fields. (c) phenomenological modelling of the Young's modulus for the longitudinal $\left(\theta=90^{\circ}\right.$ domain $)$ and transverse $\left(\theta=0^{\circ}\right.$ domain $)$ and mix oriented samples. 
Table I: Material parameters used for the numerical calculation for lead zirconate titanate (PZT) and Terfenol-D (TFD).

\begin{tabular}{|l|l|l|}
\hline & TFD & PZT \\
\hline Length $\left(10^{-3} \mathrm{~m}\right)$ & 14.5 & 14.5 \\
\hline Thickness $\left(10^{-3} \mathrm{~m}\right)$ & 0.56 & 0.127 \\
\hline Density $\left(\mathrm{Kg} / \mathrm{m}^{3}\right)$ & 9200 & 7460 \\
\hline $\mathrm{s}_{11}\left(10^{-12} \mathrm{~m}^{2} / \mathrm{N}\right)$ & 25 & 17 \\
\hline $\left.\mathrm{s}_{12} 10^{-12} \mathrm{~m}^{2} / \mathrm{N}\right)$ & -1.8 & -5.8 \\
\hline $\mathrm{s}_{13}\left(10^{-12} \mathrm{~m}^{2} / \mathrm{N}\right)$ & -16.7 & -8.8 \\
\hline $\mathrm{s}_{33}\left(10^{-12} \mathrm{~m}^{2} / \mathrm{N}\right)$ & 40 & 22.9 \\
\hline $\mathrm{d}_{31}\left(10^{-12} \mathrm{~m} / \mathrm{V}\right)$ & & -243 \\
\hline $\mathrm{d}_{33}\left(10^{-12} \mathrm{~m} / \mathrm{V}\right)$ & & 574 \\
\hline $\mathrm{q}_{31}\left(10^{-9} \mathrm{~m} / \mathrm{A}\right)$ & -13 & \\
\hline $\mathrm{q}_{33}\left(10^{-9} \mathrm{~m} / \mathrm{A}\right)$ & 26 & \\
\hline$\varepsilon_{33}$ & & 2870 \\
\hline
\end{tabular}




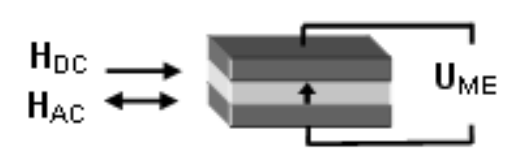

a)
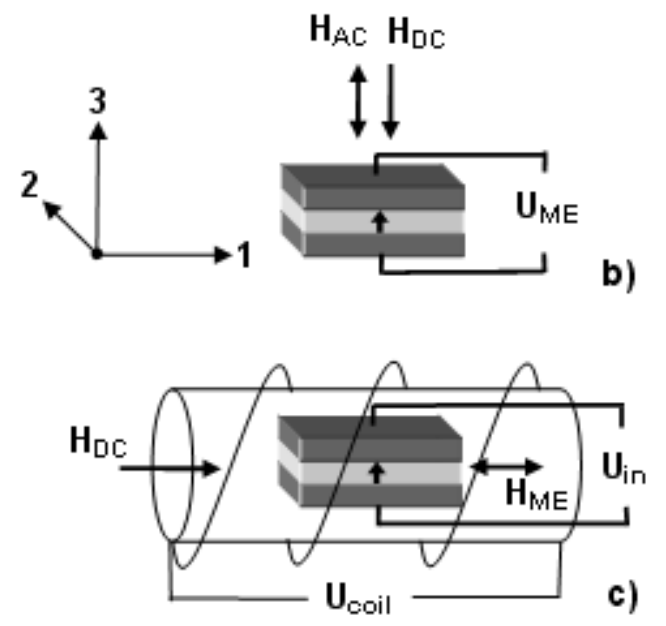

Fig. 1 

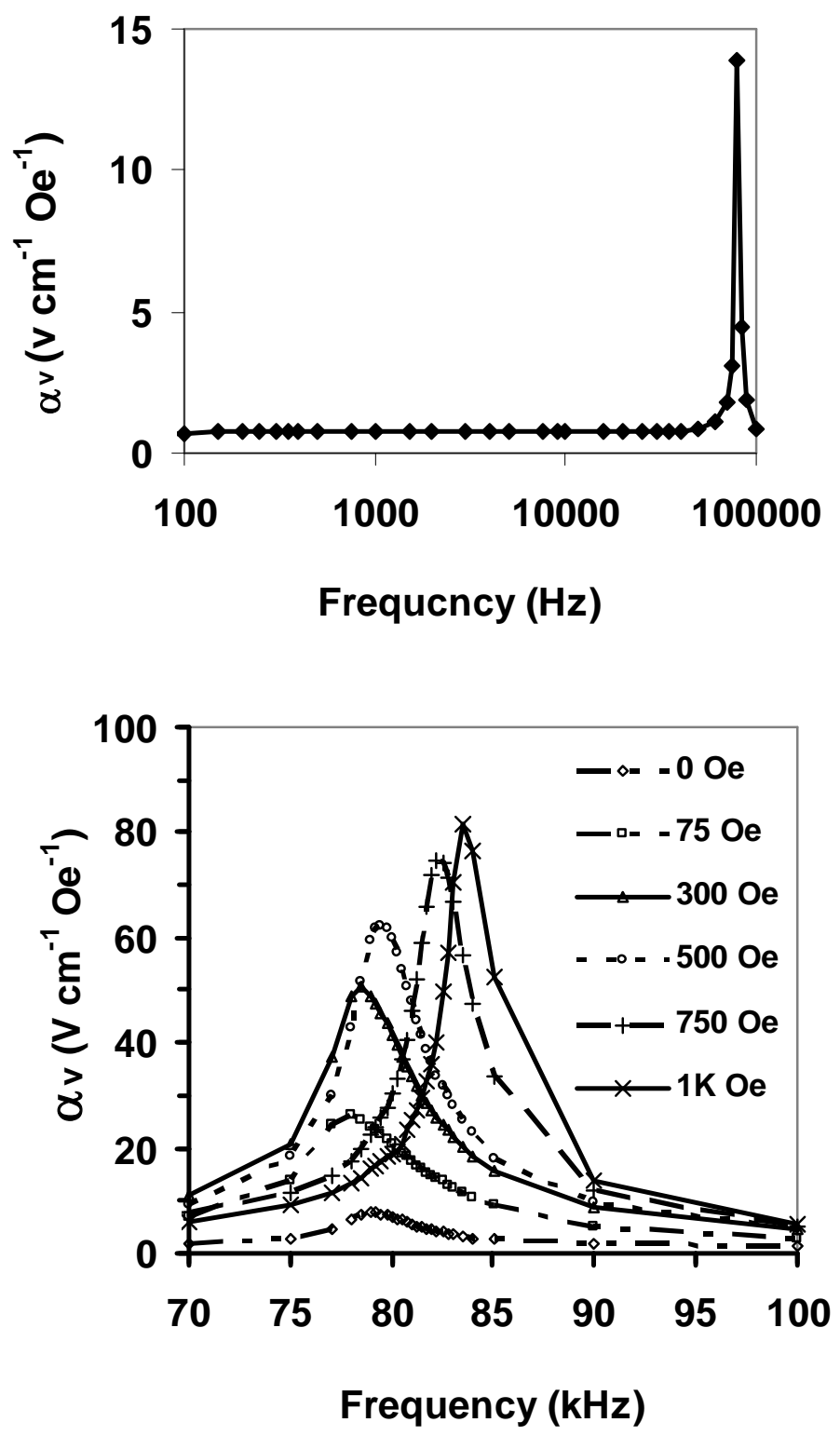

Fig. 2 


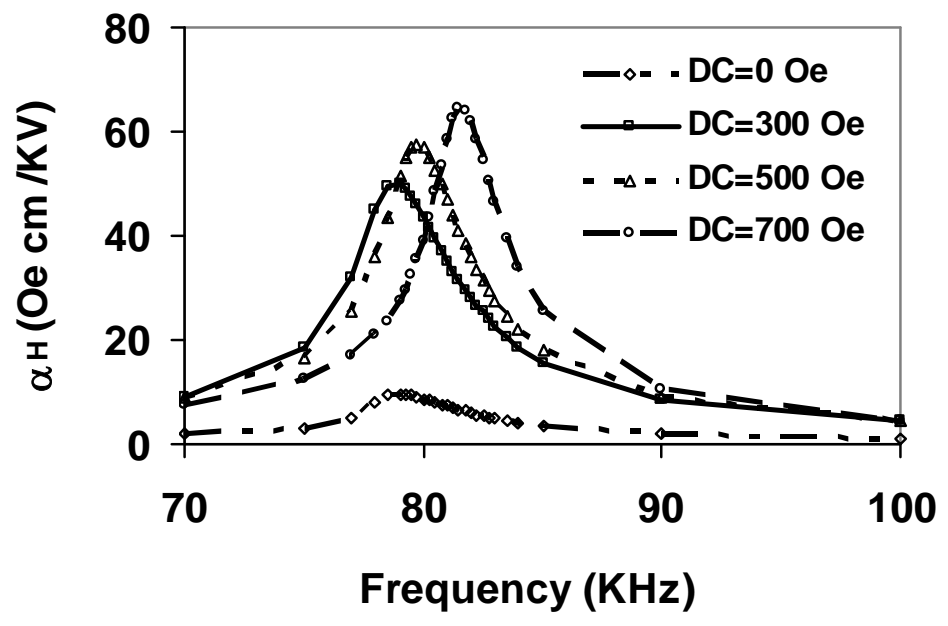

Fig. 3

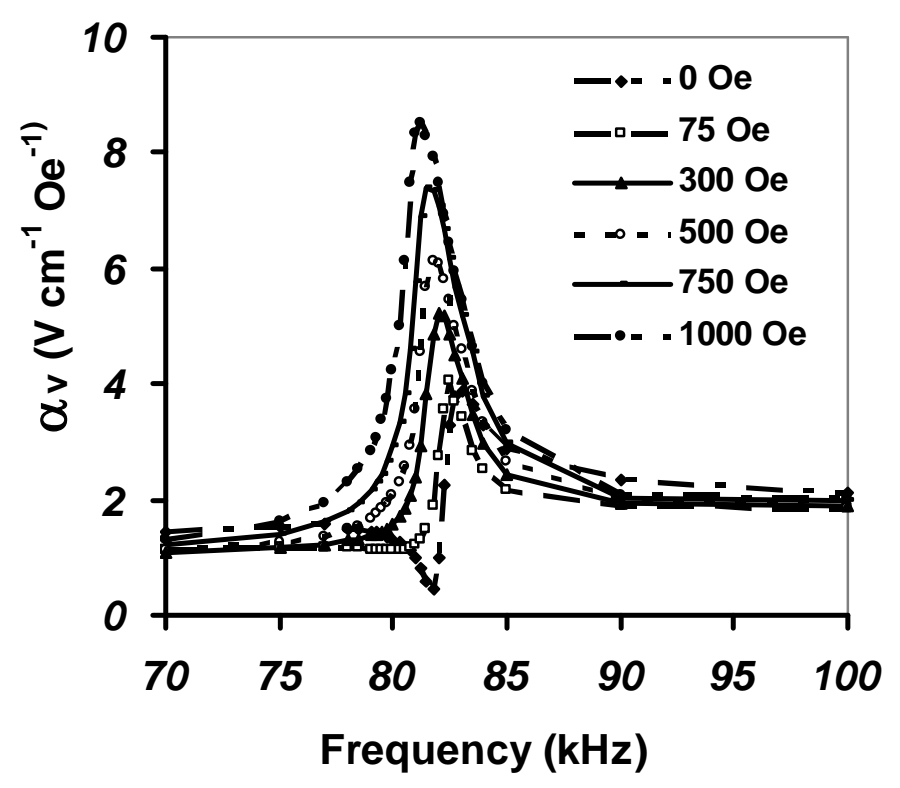

Fig. 4 

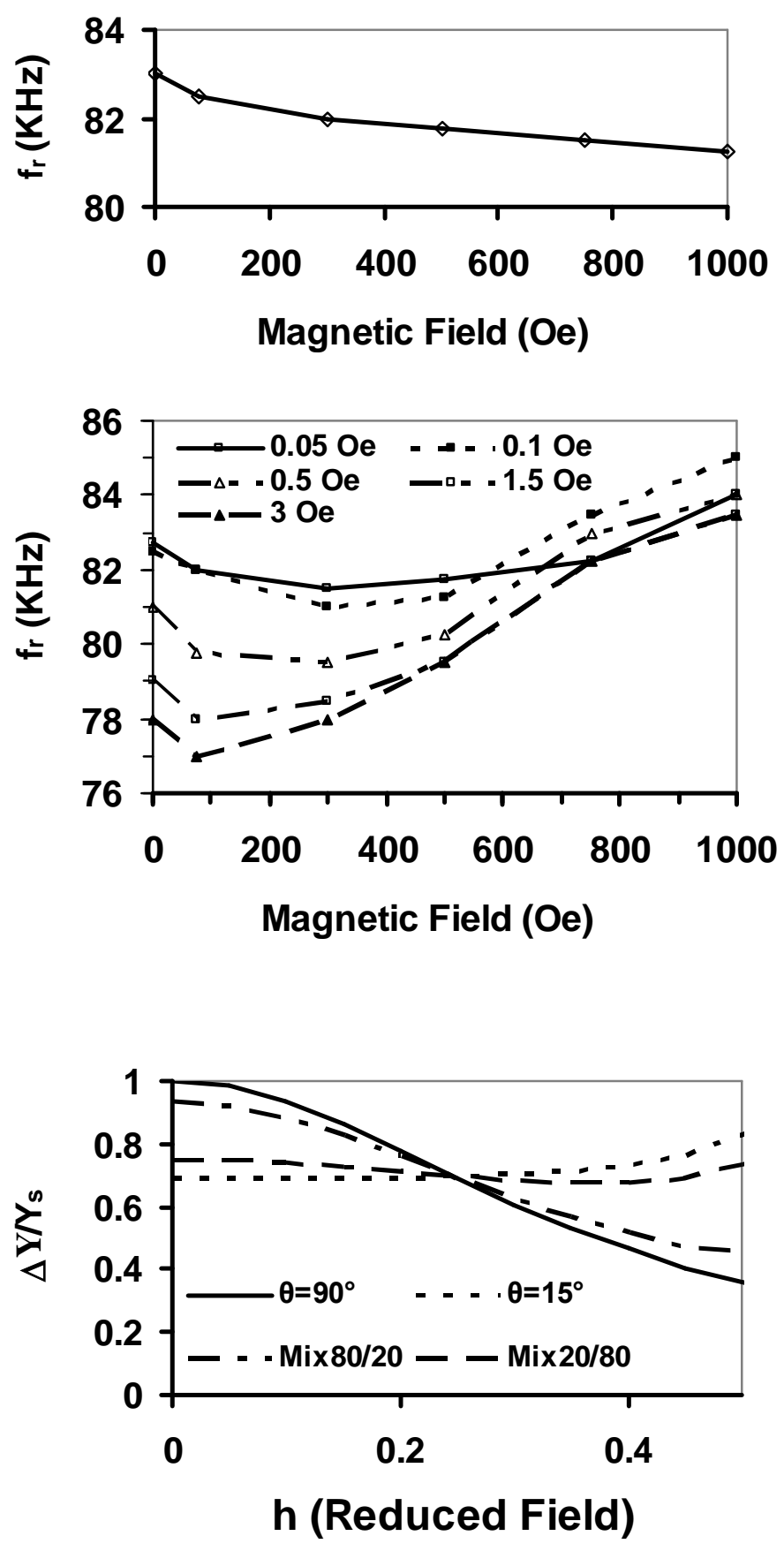

Fig. 5 